\title{
Vertebral artery hypoplasia and chronic migraine: is there an association or just an incidental finding?
}

\section{Patrick Emanuell Mesquita Sousa-Santos (D) , Gabriela Figueiredo Pucci iD , Juliana Akita}

São Paulo State University, UNESP, Botucatu, São Paulo, Brazil.

\section{$凶$}

Patrick Emanuell Mesquita Sousa-Santos

Av. Professor Montenegro s/n, Rubião Júnior, 18618-687,

Botucatu-SP-Brazil.

Email: patrickemanuell@gmail. com

\section{Edited by:}

Marcelo Moraes Valença

Keywords:

Vertebral artery

Chronic migraine

Psychiatric comorbidity

Nervous system diseases

Anatomy.

\begin{abstract}
We describe a 29-year-old woman with chronic migraine and psychiatric comorbidities that presented with new transient left-sided hemiparesis and hemi-hypoesthesia and were found to have right vertebral artery hypoplasia (VAH). We briefly review the association of VAH and migraine and the influence of psychiatric disorders and $\mathrm{VAH}$ as possible risk factors for chronification of episodic migraine. Despite uncertain mechanisms, VAH may be one of the contributing factors for the chronicity of migraine.
\end{abstract}




\section{Introduction}

V ertebral artery hypoplasia (VAH) is an uncommon variation of the posterior circulation. It was first described in the 19th century, with a prevalence varying from 2 to $6 \%$ found on autopsy and radiological exams. ' Despite this prevalence, little is known about its clinical relevance, and recently, VAH has been considered a risk factor for migraine with aura, vestibular migraine, and posterior circulation stroke. ${ }^{2}$ The frequency of VAH can be as high as four times higher in patients with migraines with aura when compared to controls. ${ }^{3}$ Moreover, few studies address the therapeutic responses in these patients. We describe a case of vertebral artery hypoplasia in a patient with chronic migraine associated with psychiatric disorders and briefly review the literature.

\section{Case Report}

A 29-year-old woman with a previous history of migraine without aura was admitted to the emergency department (ED) of a tertiary neurological center with new sudden onset of left-sided hemiparesis and hemihypoesthesia, with a duration of one minute. After these symptoms, she developed a headache with migraine characteristics, identical to her previous episodes. She denied any other previous episodes or familial history of similar symptoms.

Her previous headaches were characterized by left hemicranial, pulsatile, moderate to severe intensity, associated with nausea, vomiting, photophobia, phonophobia, and osmophobia, and have presented daily since adolescence. The duration was between 12-24 hours and used to improve with analgesic (dipyrone $500 \mathrm{mg}$ or dipyrone $300 \mathrm{mg}+$ caffeine $50 \mathrm{mg}+$ orphenadrine 35 $\mathrm{mg}$ ). She has been taking these medications more than 15 days per month in the last two years.

She also had a history of anxiety, depression, asthma, and obesity (BMI $39 \mathrm{~kg} / \mathrm{m}^{2}$ ). She denied a previous history of transient ischemic attack or stroke. In addition to simple analgesics, she used fluoxetine (40 mg per day) and combined oral contraceptive.

On ED evaluation, the neurological examination was normal. Complete metabolic panel and blood glucose were unremarkable. The headache resolved one hour after treatment with intravenous naproxen.

A brain computed tomography with perfusion and a cerebral and a cervical computed tomography angiography were requested to investigate the new neurological symptoms. Imaging exams showed a hypoplastic right vertebral artery, with no other abnormalities (Figures 1 and 2). The remaining laboratory tests, including investigation of vasculitis and other autoimmune diseases, were unremarkable.

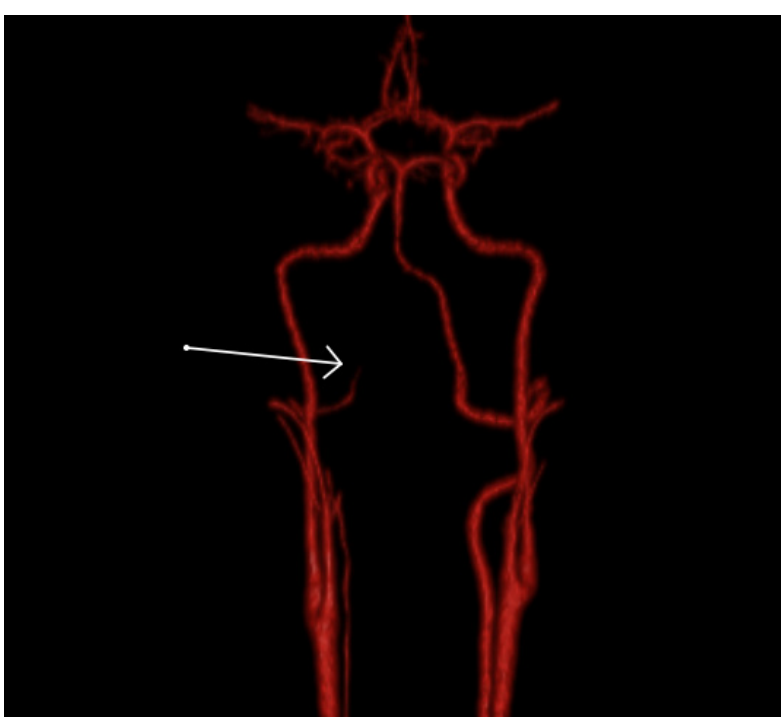

Figure 1. Digital reconstruction of cerebral and cervical CT angiography. The arrow shows the hypoplasic right vertebral artery, with little contrast filling.

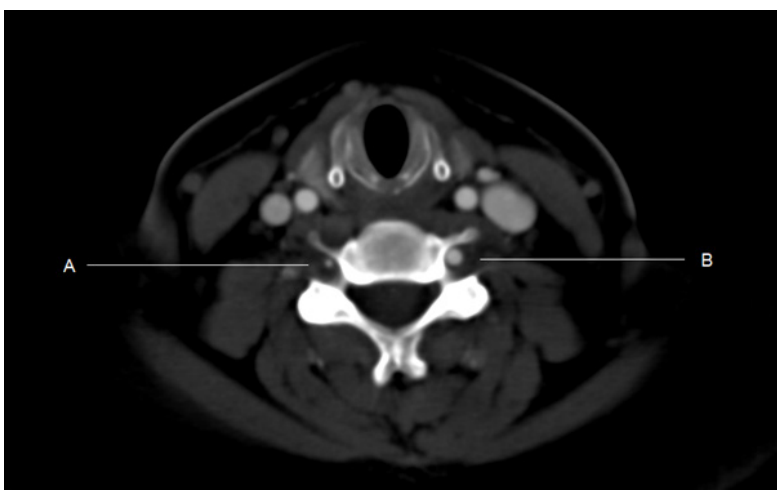

Figure 2. Axial CTA showing the difference in caliber of the right (A) and left (B) vertebral arteries.

She was diagnosed with chronic migraine and prescribed topiramate with a progressive dose increasing up to 100 $\mathrm{mg}$ per day as a preventive treatment for migraine.

\section{Discussion}

Computed tomography angiography has been proposed as a safe method as an alternative to cerebral digital 
subtraction angiography (DSA), considered the gold standard for studying cerebral circulation. However, since DSA is an invasive procedure, and the risk of developing neurological complications can be as high as $1 \%{ }^{4}$, we decided not to perform it in this patient, as no additional benefit would be obtained for her diagnosis or treatment.

An association between migraine and $\mathrm{VAH}$ emerged in a study done by Laurevic and colleagues, in 1998, which showed a prevalence of $29 \%$ of VAH in patients with migraine with aura comparing to $7 \%$ in patients with migraine without aura. In this article, a pathophysiological model was proposed, in which VAH was related to decreased posterior circulatory flow during migraine attacks, thus contributing to the manifestation of the aura. Later, Chung et al. ' found a similar prevalence of VAH in migraine with aura $(28.26 \%), 14$ times higher compared to controls. However, no decrease in vertebral artery flow was found in patients with VAH during migraine attacks.

Wherefore, the role of the VAH in migraine may not involve hypoperfusion during the attack phase but yet may contribute to migraine through complex neurovessels painproducing trigeminovascular pathways mechanisms. ${ }^{1,3}$ Thus, it is plausible that VAH could be a contributing factor to the process of chronification of episodic migraine (Figure $3)$.

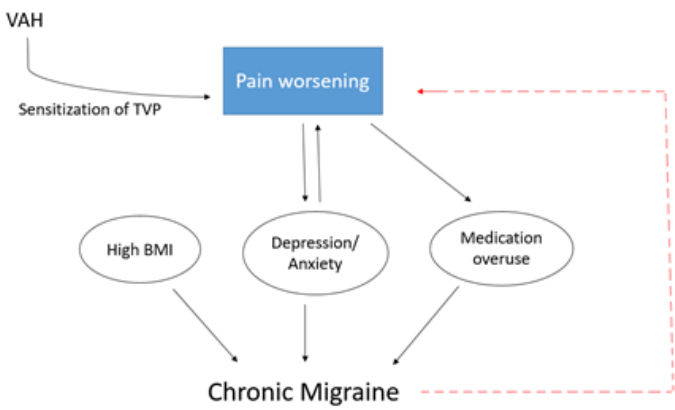

Figure 3. The role of $\mathrm{VAH}$ and Migraine. $\mathrm{VAH}$, vertebral arteria hypoplasia; TVP, trigeminovascular pathways; BMI, body mass index.

Chronic migraine $(C M)$ is defined as frequent headache attacks with at least 15 headache days per month for more than 3 months, and in at least 8 days per month with migraine characteristics. ${ }^{6}$ There are several predisposing factors to the chronification of a migraine, such as overuse of analgesics, inadequate prophylactic treatment for migraine and stressful events, high BMl, in addition to sensitization of the trigeminal system and various molecular mechanisms, such as calcitonin gene-related peptide
(CGRP) and serotonin (5-HT). ${ }^{7}$ Our patient presented some predisposing factors for $\mathrm{CM}$ associated with obesity and psychiatric comorbidities.

Therefore, management of $\mathrm{CM}$ includes a rigorous control of risk factors, acute pain relief, and effective prophylactic treatment of migraine. ${ }^{7}$ Another fundamental step in $\mathrm{CM}$ is the treatment of psychiatric disorders and other comorbidities. The overlapping symptoms of migraine, anxiety, and mood disorders make the treatment very challenging. Once the concept of migraine/tensiontype headache and depression/anxiety are continuum pathologies, it is reasonable to think of a possible spectrum or continuum between migraine and anxiety/mood disorders. ${ }^{8}$ Few studies have analyzed the most effective medications for patients with these three conditions, and a rational approach based on the main symptoms may be an option. ${ }^{8}$

Topiramate, at a daily dose of $100 \mathrm{mg}$, is generally an effective and tolerable prophylactic drug to $\mathrm{CM}^{9}{ }^{9}$ Other preventive medications, such as amitriptyline, val proate, and pregabalin, have also been shown to be effective in some studies. ${ }^{9}$ Considering pain relief, tricyclic antidepressants (amitriptyline, nortriptyline) seem to be more effective than SNRIs selective serotonin/norepinephrine reuptake inhibitor) and SSRIs (selective serotonin reuptake inhibitor). However, the treatment of anxiety and depression has better responses to SNRIs and SSRIs, and doses of tricyclic antidepressants are often higher than those used for the preventive treatment of migraine, making it difficult to be tolerated. ${ }^{10}$ In our patient, we chose to start topiramate for migraine prophylaxis and maintain fluoxetine, as she showed an adequate response to anxiety and depression.

\section{Conclusion}

Despite the role of $\mathrm{VAH}$ in migraine being unclear, $\mathrm{VAH}$ may contribute to the chronification of migraine through complex pain-producing trigeminovascular pathways mechanisms. However, it is important to be aware of factors such as overuse of analgesics and psychiatry comorbidities for the adequate treatment of chronic migraine.

Declaration of conflicting interests: The authors declared no potential conflicts of interest with respect to the research, authorship, and/or publication of this article.

Funding: The authors received no financial support for the research, authorship, and/or publication of this article.

Author's Contribution: PEMSS, conceptualization, data curation, writing - original draft; GFP, data curation, 
writing - review \& editing; JA, supervision, data curation, writing - review \& editing.

Patrick Emanuell Mesquita Sousa-Santos https://orcid.org/0000-0002-7729-7303

Gabriela Figueiredo Pucci

https://orcid.org/0000-0002-6597-6106

Juliana Akita

https://orcid.org/0000-0002-2645-5543

\section{References}

1. Chuang YM, Chan L, Wu HM, Lee SP and Chu YT. The clinical relevance of vertebral artery hypoplasia. Acta Neurol Taiwan 2012;21(1):1-7

2. Chen JJ, Chang HF, Chen DL and Tseng CH. Which Vestibulopathy is Vertebral Artery Hypoplasia Related with in Vestibular Migraine? Acta Neurol Taiwan 2015;24(1):1-10

3. Chuang YM, Hwang YC, Lin CP and Liu CY. Toward a further elucidation: role of vertebral artery hypoplasia in migraine with aura. Eur Neurol 2008;59(3-4): 148 151 Doi:10.1159/000111878

4. Alakbarzade V and Pereira AC. Cerebral catheter angiography and its complications. Pract Neurol 2018;18(5):393-398 Doi:10.1136/ practneurol-2018-001986
5. Lovrencić-Huzjan A, Demarin V, Rundek T and Vuković $\mathrm{V}$. Role of vertebral artery hypoplasia in migraine. Cephalalgia 1998;18(10):684-686 Doi:10.1046/ j. 1468-2982.1998.1810684.x

6. International Headache Society. Headache Classification Committee of the International Headache Society (IHS) The International Classification of Headache Disorders, 3rd edition. Cephalalgia 2018;38(1):1-211 Doi: $10.1177 / 0333102417738202$

7. Su $M$ and $Y_{U}$ S. Chronic migraine: A process of dysmodulation and sensitization. Mol Pain 2018;14:1744806918767697 Doi:10.1177/1744806918767697

8. Peres MFP, Valença MM and Silva-Neto RP. Management of psychiatric comorbidities in migraine. Headache Medicine 2018;9(2):61-67 Doi:10.48208/HeadacheMed.2018.13

9. Diener $H C$, Bussone $G$, Van Oene JC, Lahaye $M$, Schwalen S and Goadsby PJ. Topiramate reduces headache days in chronic migraine: a randomized, double-blind, placebo-controlled study. Cephalalgia 2007; 27 (7): 814-823 Doi: 10.1111/j.14682982.2007.01326.x

10. Torta $R$ and leraci V. Migraine and depression comorbidity: antidepressant options. Neurol Sci 2012;33 Suppl 1:S117-118 Doi:10.1007/s10072012-1055-4 